\title{
Double Chooz status
}

\author{
Junpei Maeda*† \\ Department of Physics, Tokyo Metropolitan University, Tokyo, Japan \\ E-mail: maeda@hepmail.phys.se.tmu.ac.jp
}

Double Chooz is a neutrino oscillation experiment that will measure the last undetermined mixing angle $\theta_{13}$ or improve the current best limit, using reactor neutrinos at the Chooz nuclear power plant in France. The detector has been constructed since 2009, and being filled with liquid scintillator since October 2010. The data taking will start from the beginning of 2011, and the current upper limit of $\sin ^{2} 2 \theta_{13}$ will be surpassed by summer of 2011, using data taken during a first run period. This paper presents the experimental concept and the status of the Double Chooz experiment, which aims at measuring the $\theta_{13}$ mixing angle with an expected sensitivity $\sin ^{2} 2 \theta_{13}<0.03$ at $90 \%$ C.L., for $\Delta m_{31}^{2}=2.5 \times 10^{-3} \mathrm{eV}^{2}$.

The Xth Nicola Cabibbo International Conference on Heavy Quarks and Leptons, October 11-15, 2010

Frascati (Rome) Italy

\footnotetext{
* Speaker.

$\dagger$ on behalf of the Double Chooz collaboration
} 


\section{Introduction}

Neutrino oscillation proves the mass difference between two neutrino flavours, and it can be described by the Maki-Nakagawa-Sakata (MNS) matrix. In the matrix, two mixing angles, $\theta_{12}$ and $\theta_{23}$, were measured by recent experiments, and they are fairly large [1]. On the other hand, only upper limit is set to the last mixing angle $\theta_{13}, \sin ^{2} 2 \theta_{13}<0.15$, and it is very small compared to other mixing parameters. Hence $\theta_{13}$ value may play a key role when building the unified theory, and finite value of $\theta_{13}$ leads us to the next step of neutrino experiments, such as to measure $\delta_{C P}$ in neutrino sector and mass hierarchy. Therefore, the current important step of neutrino physics is to measure the $\theta_{13}$ value or to set a stringent upper limit.

The reactor neutrino disappearance experiments are a sensitive probe of $\theta_{13}$. The past $\mathrm{CHOOZ}$ experiment [2] set the current best limit of $\sin ^{2} 2 \theta_{13}<0.15$ at $90 \%$ C.L. for $\Delta m_{31}^{2}=2.5 \times 10^{-3} \mathrm{eV}^{2}$. Reactor neutrino spectrum is going up to $10 \mathrm{MeV}$, thus the inverse beta decay, $\bar{v}_{e}+p \rightarrow e^{+}+n$ shows the highest cross section and a spectrum peaked around $4 \mathrm{MeV}$. Thereby at the $1 \mathrm{~km}$ baseline, simple 2 flavour oscillation formula is valid and is well suited for a maximal sensitivity to $\theta_{13}$ free of parameters degeneracies. The survival probability of $\bar{v}_{e}$ is well approximated by:

$$
P\left(\bar{v}_{e} \rightarrow \bar{v}_{e}\right)=1-\sin ^{2} 2 \theta_{13} \sin ^{2}\left(\frac{1.27 \Delta m_{31}^{2} L}{E}\right)+O\left(10^{-3}\right)
$$

where $\Delta m_{31}^{2}=m_{3}^{2}-m_{1}^{2}$ is in $\mathrm{eV}^{2}, L$ is the source to detector distance in metres and $E$ is the $\bar{v}_{e}$ energy in MeV. Since the $\Delta m_{31}^{2}$ is well known, matter effects are negligible, and it is independent of CP-violation phase, the reactor neutrino experiment can measure $\theta_{13}$ within the small physical uncertainties. The Double Chooz experiment [3] inherits the CHOOZ experiment concept with many improvements on both systematic and statistic errors.

\section{The concept of Double Chooz}

The Double Chooz detectors are located at the Chooz nuclear power station in the Ardennes region in France. This site contains two pressurised water reactors ( $4.37 \mathrm{GW}$ each).

Anti-electron neutrinos from the reactors interact with protons through inverse beta decays in the Double Chooz detector. The produced positron emits scintillation light while running and eventually annihilates with an electron instantly, called "prompt signal", and the neutron will be thermalised, then captured by Gadolinium in target region. After the neutron capture, Gadolinium releases some gamma-rays whose energy is $8 \mathrm{MeV}$ in total, called delayed signal. The time interval between the two signals is about $30 \mu \mathrm{s}$. A neutrino signal will be identified by these two signals, called delayed coincidence, and backgrounds can be significantly suppressed by requiring this coincidence. At the far detector, about 70 neutrino signals per day are estimated. The visible energy deposition $E_{v i s}$ due to the $e^{+}$is related to the $\bar{v}_{e}$ energy $E_{v}: E_{v}=E_{v i s}+0.8 \mathrm{MeV}$.

The signals may be contaminated by background events which can be divided into two classes called accidental and correlated. Accidental backgrounds are produced by an accidental coincidence with environmental gamma-rays from detector material and rocks, while the correlated ones are caused by one process, such as a fast neutron induced by cosmic-rays. At the Double Chooz far detector, each background is estimated as $\sim 2$ events/day and $\sim 1.6$ events/day, respectively. 
The previous $\mathrm{CHOOZ}$ experiment took data only during a few months due to degradation of the $5.6 \mathrm{~m}^{3}$ of target liquid scintillator, the statistical error hence amounted to $2.8 \%$. The Double Chooz experiment has thus enlarged the target volume to $10.3 \mathrm{~m}^{3}$ and developed a new scintillator composite to reach a stability of at least 3 years for a statistical error down to $0.5 \%$. The CHOOZ systematic error amounted to $2.7 \%$ with contributions due to the lack of knowledge of the $\bar{v}_{e}$ source (reactor fuel cross section, reactor power and $\bar{v}_{e}$ per fission), on the number of target protons and on the detection efficiency. In order to reduce systematic errors, Double Chooz has two identical detectors, near and far detector. The reactor-induced errors which are the largest contribution in systematic uncertainties can be reduced by performing a relative measurement. The disappearance of reactor neutrinos are measured using the far detector, and the near detector will be used for neutrino flux measurement. The near detector is placed in the vicinity of the cores at a mean distance of $400 \mathrm{~m}$ with an overburden of 120 m.w.e. In the far detector, located at $1050 \mathrm{~m}$ and a depth of 300 m.w.e., we look for a departure of the expected number of $\bar{v}_{e}$ from the $1 / L^{2}$ evolution.

\subsection{Detector design}

The far detector is located in the old CHOOZ laboratory. The dimensions were constrainted to a cylinder of $7 \mathrm{~m}$ in diameter and height. It is made of concentric layers as can be seen on Fig. 1 .

The innermost volume ('target') is Gd-doped $10.3 \mathrm{~m}^{3}$ liquid scintillator in an acrylic vessel. The use of $\mathrm{Gd}$ allows to have an $8 \mathrm{MeV}$ gamma-ray cascade induced by the neutron capture typically $30 \mu$ s after the $e^{+}$energy deposition and annihilation. This scintillator was newly developed to achieve a long term stability. The target volume is contained in a $55 \mathrm{~cm}$ thick non Gd-doped liquid scintillator called ' $\gamma$-catcher' whose purpose is to contain the signal gamma-rays and to have

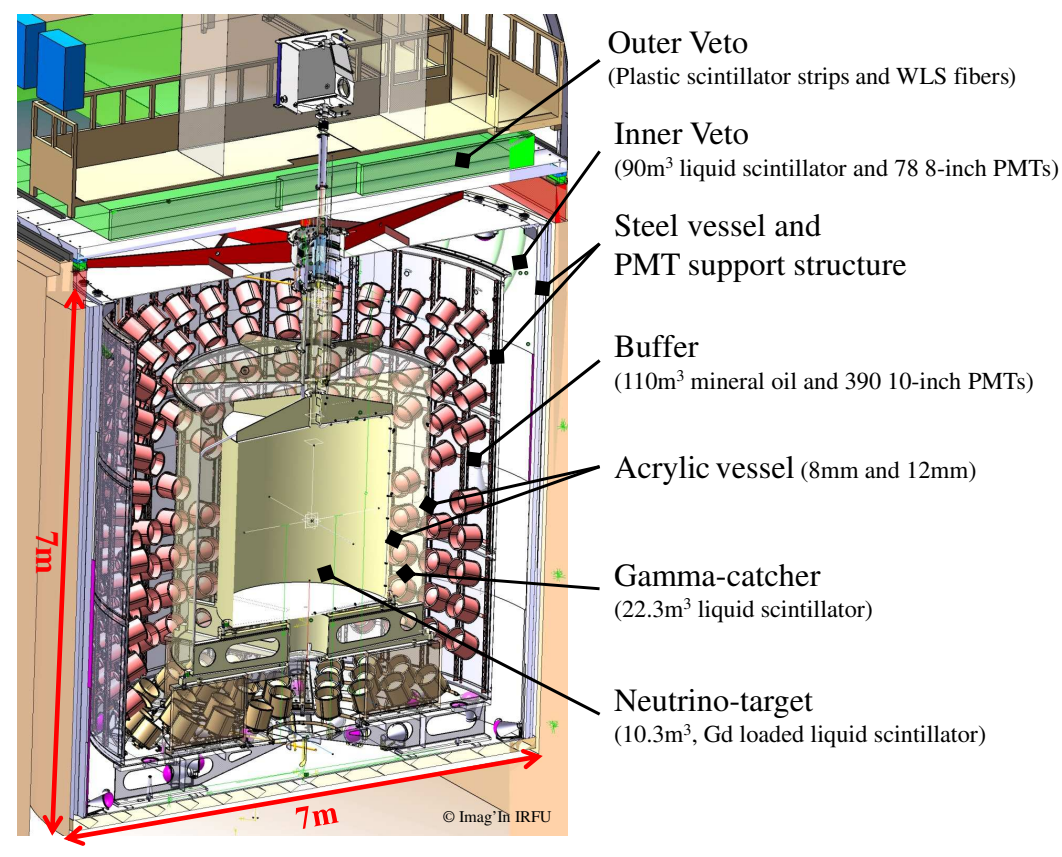

Figure 1: Design of the Double Chooz detector. 
a precisely defined target volume. The light yield of $\gamma$-catcher scintillator is the same as Gd-doped scintillator in the target region. A $105 \mathrm{~cm}$ thick non scintillating volume contained in a steel vessel ('buffer') surrounds the previous detection volumes. The stainless steel vessel of $3 \mathrm{~mm}$ thick holds 390 low background 10" photomultiplier tubes (PMTs) for a 13\% photocathode coverage, to detect the light from the central scintillating regions. The non-scintillating oil in the buffer region can attenuate the gamma-rays emitted from the PMTs radioactivity.

The next volume called 'inner veto' (IV) is a $50 \mathrm{~cm}$ thick liquid scintillator volume contained in a steel vessel. It is optically separated from the other regions and holds 78 8" PMTs. This voume is complementary to the 'outer veto' (OV) that is made of plastic scintillator strips with wavelength shifting fibers and multi-anode PMTs disposed orthogonally and covering the detector top surface with a few meters extension. The purpose of these two volumes is to estimate the amount of the correlated background induced by cosmic-ray muons. The last concentric volume is a $15 \mathrm{~cm}$ thick steel shielding against the rock radioactivity. Together with the buffer, it allows to reduce by two orders of magnitudes of the accidental background with resepct to the $\mathrm{CHOOZ}$ experiment.

\subsection{Sensitivity and potential}

As already mentioned, the statistical error will be reduced to be less than $0.5 \%$, by increasing the target volume from the $\mathrm{CHOOZ}$ experiment and by a longer data taking period. Moreover, since both detectors are identical and the number of cuts needed at analysis level is reduced thanks to a better detector design, many systematic uncertainties will be canceled out as shown in Tab. 1 . The Double Chooz overall systematic error is expected to be less than $0.5 \%$.

The Double Chooz experiment will have two different phases: the phase I when the far detector will be running alone and the phase II when both detectors will be running simultaneously, about 1.5 years after the beginning of the phase I. Figure 2 shows the $\sin ^{2} 2 \theta_{13}$ limit at $90 \%$ C.L. for $\Delta m_{13}^{2}=2.5 \times 10^{-3} \mathrm{eV}^{2}$ in case of no oscillation observation as a function of time. The current limit

\begin{tabular}{l|l|c|c}
\hline \multirow{2}{*}{$\begin{array}{l}\text { Reactor } \\
\text { neutrino flux })\end{array}$} & & CHOOZ & Double Chooz \\
\cline { 2 - 4 } & Production x-sec & $1.9 \%$ & - \\
\cline { 2 - 4 } & Reactor power & $0.7 \%$ & - \\
\cline { 2 - 4 } & Energy per fission & $0.6 \%$ & - \\
\cline { 2 - 4 } Detector & Dolid angle & - & $0.1 \%$ \\
\cline { 2 - 4 } & Tetection x-sec & $0.3 \%$ & - \\
\cline { 2 - 4 } & Fidget mass & $0.3 \%$ & $0.2 \%$ \\
\cline { 2 - 4 } & H/C ratio & $0.2 \%$ & - \\
\cline { 2 - 4 } & Dead time & $0.8 \%$ & - \\
\hline Analysis & Selection efficiency & $1.4 \%$ & - \\
\hline \hline \multicolumn{2}{c}{ Total systematic error } & $2.7 \%$ & $<0.4 \%$ \\
\hline \multicolumn{2}{c}{ Statistical error } & $2.8 \%$ & $<0.5 \%$ \\
\hline
\end{tabular}

Table 1: Systematic and statistic errors compared between CHOOZ and Double Chooz experiments [4]. The hyphen denotes the uncertainty is negligible. 
from $\mathrm{CHOOZ}$ experiment will be surpassed quickly at the beginning of phase I. Then the sensitivity saturates due to the systematic error around $\sin ^{2} 2 \theta_{13} \leq 0.06$ at the end of phase I. In the phase II, we enter a second systematic error regime, and after 3 years a sensitivity of $\sin ^{2} 2 \theta_{13} \leq 0.03$ can be reached. The discovery potential is $\sin ^{2} 2 \theta_{13} \geq 0.05$ at $3 \sigma$ after 3 years with both detectors running. A global analysis using Solar and KamLAND data predicts $\sin ^{2} 2 \theta_{13}=0.08_{-0.07}^{+0.08}$ [5]. There is a reasonable chance to make the measurement of non-zero $\theta_{13}$ in Double Chooz.

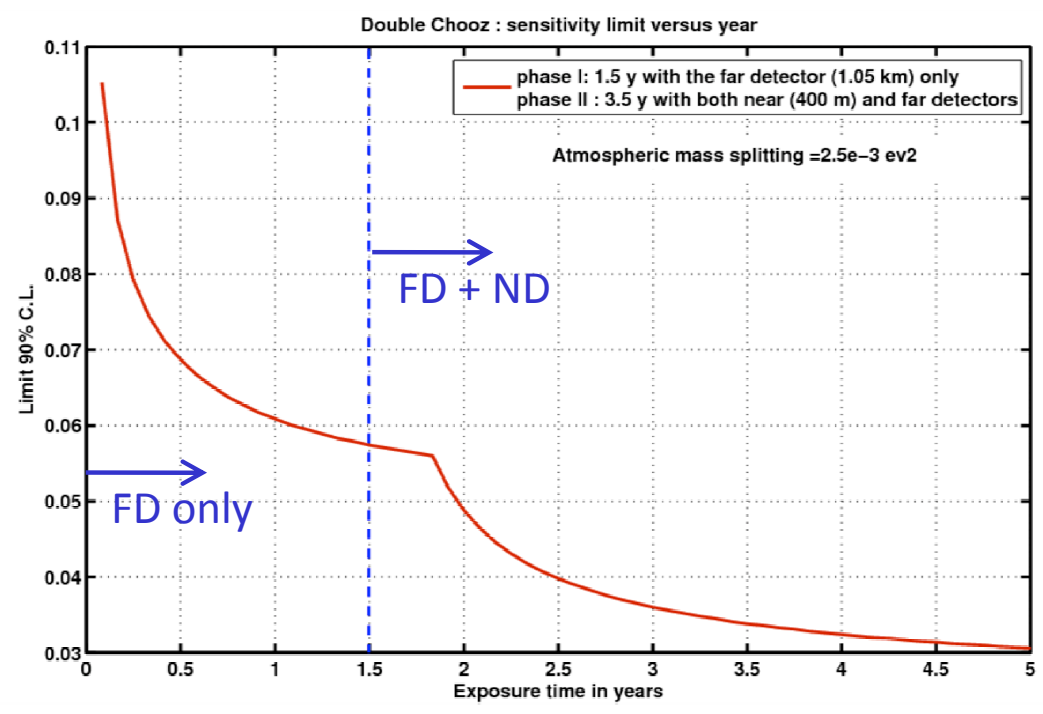

Figure 2: $\sin ^{2} 2 \theta_{13}$ sensitivity at $90 \%$ C.L. as a function of time for $\Delta m_{31}^{2}=2.5 \times 10^{-3} \mathrm{eV}^{2}$, assuming that the near detector running is started 1.5 years after the start of the far detector operation.

\section{Current status of the Double Chooz experiment}

Each Double Chooz detector contains 390 PMTs in buffer region. After individual test of PMTs, those on the bottom and side wall were installed in summer 2009. After that, acrylic vessels were installed. The installation took place in three steps: the $\gamma$-catcher was installed first; then the target vessel was installed in the $\gamma$-catcher; the lid part of vessel was glued as the last step (Fig. 3).

Each Double Chooz detector will contain $237 \mathrm{~m}^{3}$ of liquids. All liquids have been prepared and stored in the storage area which is located in the far detector site. After installation of the detector filling operation system, the nitrogen flushing was done. The detector has started the liquid filling since October 2010, and will be finished in December 2010. In parallel, the DAQ and electronics commissioning with data taking of so-called dry-detector is ongoing. The first signal from PMTs was taken in June 2010, and first neutrino data are expected from January 2011.

For the near detector, the civil work will start at the beginning of 2011, and the laboratory will be available by the end of 2011 to start the installation. Both, near and far detectors, will be operative by mid 2012. 


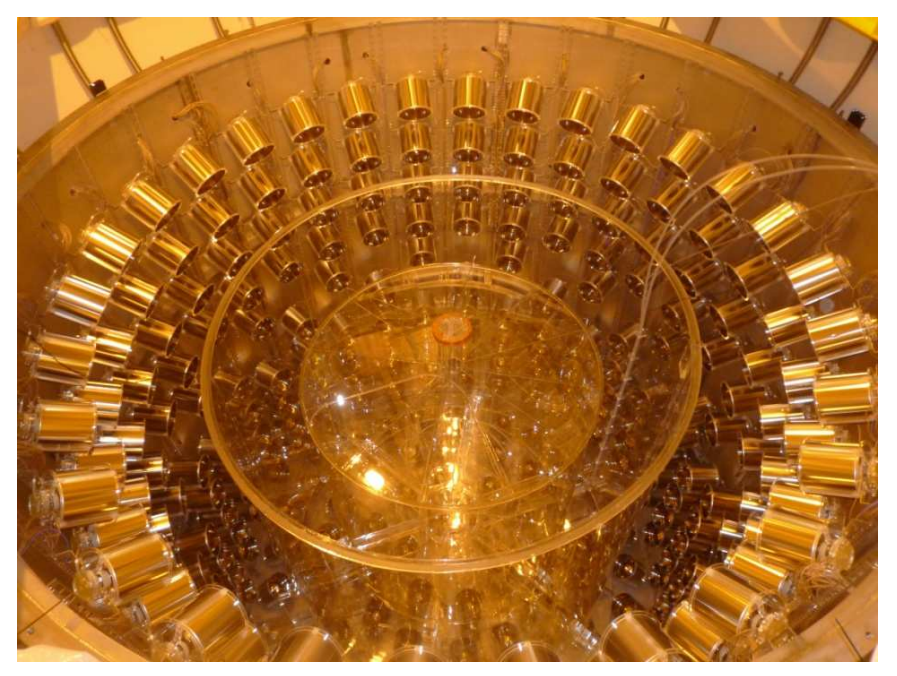

Figure 3: View of the acrylic vessels inside the buffer tank with the PMTs.

\section{Conclusion}

Double Chooz is a coming reactor neutrino experiment using two identical detectors at different distances to measure the still undetermined mixing angle $\theta_{13}$. Double Chooz has almost completed the installation of the far detector, and the filling of liquid scintillator is ongoing. It is expected to start data taking at the beginning of 2011. The near detector will be ready in 2012 for the second phase of the experiment. After three years of operation with both detectors, Double Chooz will be able to measure $\sin ^{2} 2 \theta_{13}$ to $3 \sigma$ if $\sin ^{2} 2 \theta_{13}>0.05$, or put the stringent upper limit of $\sin ^{2} 2 \theta_{13}<0.03$ at $90 \%$ C.L. if no oscillation is observed.

\section{References}

[1] S. Fukuda et al., Phys. Lett. B 539 (2002) 179-187 [hep-ex/ 0205075 ].

S. Abe et al., Phys. Rev. Lett. 100 (2008) 221803.

Q. R. Ahmad et al., Phys. Rev. Lett. 89, No. 1 (2002) 011301.

[2] M. Apollonio et al., Eur. Phys. J. C 27 (2003) 331-374 [hep-ex/ 0301017 ].

[3] F. Ardellier et al., June 2006, hep-ex/0606025.

[4] G.Mention et al., April 2007, hep-ex/0704.0498.

[5] B. Aharmim et al., Phys. Rev. C 81 (2010) 055504. 